\title{
ARTICLE
}

\section{Development of a new simulation software system to evaluate radiation doses and facilitate decontamination tasks in reactor buildings}

\author{
Takuya Takahashi*, Yuji Nemoto, Hideo Nakano and Toshihisa Tsukiyama \\ Hitachi-GE Nuclear Energy, Ltd., Saiwai-cho, 3-1-1, Hitachi-shi, Ibaraki-ken, 317-0073, Japan
}

\begin{abstract}
Exploring a reasonable way to decontaminate the reactor buildings at the Fukushima Daiichi Nuclear Power Plant, a new simulation system calculating the doses originated in the contamination on the surface of structures was developed. This simulation system, called "RaF-MAP (Radiation Field MAP)", calculates doses based on built-in attenuation data conducted using MCNP5 within a short time. This paper describes the system principle of RaF-MAP, verification by comparing with doses conducted with existing code, and a comparison with the measured doses in the reactor building at Fukushima Daiichi Nuclear Power Plant. In addition, this paper shows how RaF-MAP contributes to decontamination tasks, by presenting procedures to use RaF-MAP and the dose-reduction effect provided by the hypothetical decontamination operations.
\end{abstract}

Keywords: decontamination; Monte Carlo; MCNP5; shielding design; dose distributions; reactor building; Fukushima Daiichi Nuclear Power Plant

\section{Introduction}

As the first step toward effectively facilitating decontamination tasks in the reactor buildings at Fukushima Daiichi Nuclear Power Plant (hereinafter referred to as $1 \mathrm{~F}$ ), it is imperative to understand the actual conditions, namely where and how floors and walls are contaminated by radioactive substances. Meeting the above needs, the radiation doses must be measured exhaustively at the surface of structures and in air. However, some points are difficult to measure due to the operational constraint of robot. In the case simulating the present situation of reactor buildings at the $1 \mathrm{~F}$ site with existing calculation codes, simulating widespread sources requires immense time and effort.

Therefore, a simulation system called "RaF-MAP" (Radiation Field MAP), equipped with the following functions, was developed to fill the gap in measured data:

- Obtaining highly accurate results (doses at points in air) within a short time

- Evaluating the dose-reduction effect of the decontamination operation

- Evaluating the dose-reduction effect of installing movable shields

\section{System principle of RaF-MAP}

\subsection{Basic formula of RaF-MAP}

RaF-Map is a simulation system to calculate dose in air: $D_{s}$ based on Eq. (1) using with built-in attenuation data calculated using MCNP5 [1] and measured doses on the surface of structures as input data. The structures such as floors, walls and ceilings are modeled by a combination of cubes $1 \mathrm{~m}$ on a side. The doses measured on the surfaces of structures are entered to meshes formed by a $1 \mathrm{~m} \times 1 \mathrm{~m}$ square of the input sheet in ' $\mathrm{mSv} / \mathrm{h}$ ' units as radiation sources. Figure $\mathbf{1}$ shows the illustration of the sample calculation model simulating inner contaminated building. RaF-MAP can generate contour maps showing radiation levels at an arbitrary plane as output data.

$A_{i}, R_{i}$ and $C$ are built-in data in RaF-MAP and the calculation procedures used to develop them are shown in the next sections.

$$
\left.D_{s}=\sum_{i}\left[\left(D_{m}\right)_{i} \times A_{i} \times R_{i} \times C\right)\right]
$$

$\left(D_{m}\right)_{i}$ : Measured dose rate at the surface of structures $A_{i}$ : Attenuation rate in air corresponding to $L_{i}$ $R_{i}$ : Attenuation rate in shields $C$ : Corrective coefficient with consideration of the dose contribution from the sources except Source ${ }_{i}$

\footnotetext{
* Corresponding author. Email: takuya.takahashi.cp@hitachi.com
} 


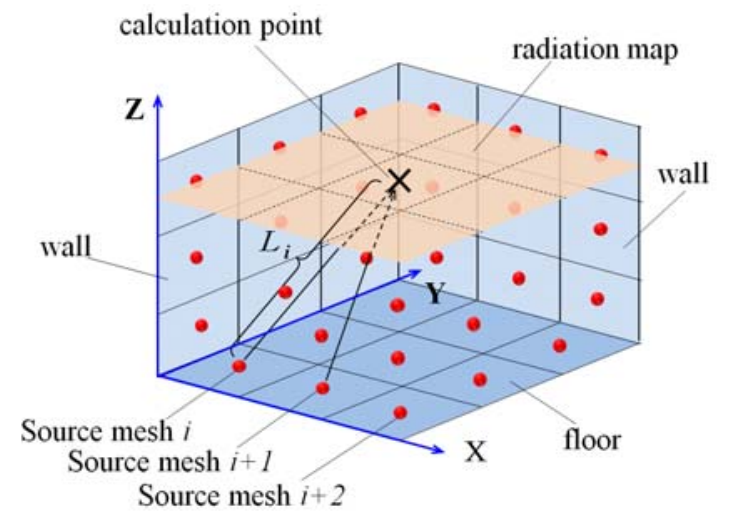

Figure 1. The illustration of inner contaminated building.

\subsection{Built-in data in RaF-MAP}

(a) Attenuation rate in the air; $A(L)$

The basic data used to determine the attenuation rate in the air were prepared by calculations under the conditions listed in Table $\mathbf{1}$ and the model shown in Figure 2, whereupon the function $a(L)$ was defined by Eq. (2). To have calculation time as short as possible, calculation points in figure 2 were set only vertical direction to the plane source and this means $a(L)$ has no direction dependency.

While the function $a(L)$ is a discrete function since the calculation point of the basic data is a discrete value, the built-in data of the attenuation rate in the air, $A(L)$, needs to be a continuous function. Therefore, $a(L)$ was approximated as $A(L)$ as shown in Eq. (3) by setting invariables such that the differences between $a(L)$ and $A(L)$ are within $\pm 5 \%$. Eq. (3) was proposed by Harima in reference [2].

$$
\begin{array}{r}
a(L)=(\text { dose rate at } L \text { distance from the source }) \\
/(\text { dose rate at the surface of the source })
\end{array}
$$

$$
A(L)=K \times e^{-\alpha-\beta \times L} \times L^{-\gamma-\varepsilon \times L}
$$

Table 1. Calculation conditions used to develop $A_{i}$.

\begin{tabular}{|l|l|}
\hline Calculation code & MCNP5 \\
\hline Shape of source & $\begin{array}{l}1 \mathrm{~m} \times 1 \mathrm{~m} \text { square plane source whose } \\
\text { strength is homogeneously distributed }\end{array}$ \\
\hline Radionuclide & $\begin{array}{l}4 \text { types } \\
\text { (Cs-134,Cs137, Co-60,1 MeV } \gamma \text {-ray) }\end{array}$ \\
\hline Calculation point & $\begin{array}{l}0 \text { to } 100 \mathrm{~m} \text { point perpendicular to the } \\
\text { plane source, at } 10 \mathrm{~m} \text { interval }\end{array}$ \\
\hline
\end{tabular}

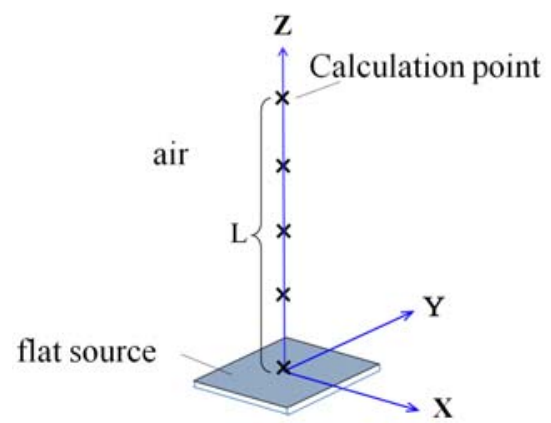

Figure 2. Calculation model used to $\operatorname{develop} A_{i}$. (b) Attenuation rate in shields: $R(t)$

The basic data used for developing the attenuation rate in shields were prepared by calculations under the conditions listed in Table 2 and the model shown in Figure 3, whereupon the function $R(t)$ was defined by Eq. (4).

$\mathrm{R}(\mathrm{t})=($ dose at the point $t$ distance from the center in Fig. 3(a)) / (dose at the point $t$ distance from the center in Fig. 3(b))

Table 2. Calculation conditions to develop $R_{i}$.

\begin{tabular}{|l|l|}
\hline Calculation code & MCNP5 \\
\hline Shape of source & Point source \\
\hline Radionuclide & $\begin{array}{l}4 \text { types } \\
\text { (Cs-134, Cs137, Co-60, } 1 \mathrm{MeV} \gamma \text {-ray) }\end{array}$ \\
\hline Shields & 2 types (concrete, steel) \\
\hline
\end{tabular}

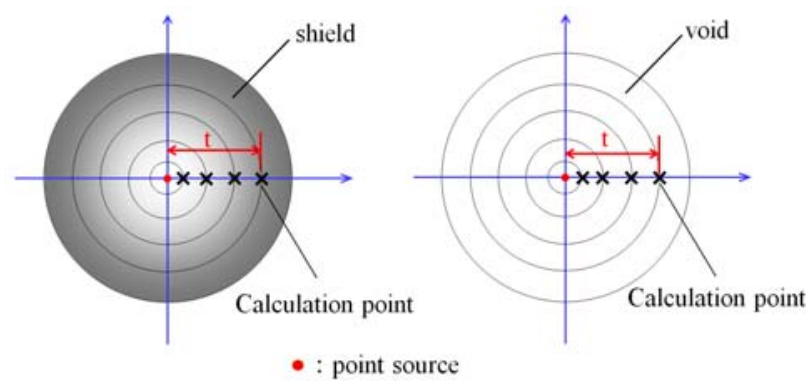

$\begin{array}{ll}\text { (a) Shield sphere model } & \text { (b) Void sphere model }\end{array}$ Figure 3. Calculation model used to develop $R_{i}$.

(c) Corrective coefficient with consideration of the dose contribution from sources except the nearest mesh: $C$

Measured doses on the surface mainly consist of the dose contributions from the nearest floor contamination but also include the contributions located away from the measurement point. If the measured doses on the surface are entered to all corresponding meshes in the input sheet, the calculated values on the surface obviously exceed the entered measured doses. This discrepancy is attributable to contribution from sources except the nearest mesh to the calculation point shown in Figure 4.

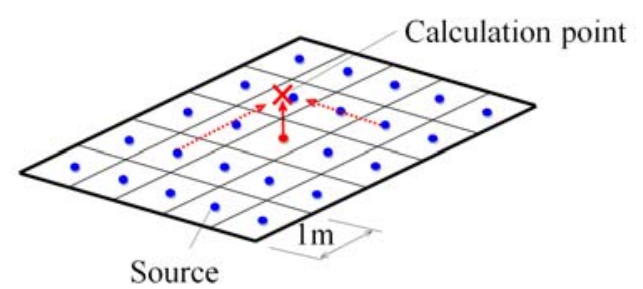

Figure 4. Consideration of the dose contribution from the sources except the nearest mesh.

To enter the raw measured doses without the discrepancy mentioned above, a corrective coefficient; $C$ is built into RaF-MAP. C is a variable number varies depend on the height of the measured point from the source surface. The basic data used for developing $C$ were prepared by calculating under the conditions listed in Table 3. For example, If doses on the surface of structures are measured at $1 \mathrm{~cm}$ point from the surface, $C$ 
is defined by Eq. (5) with the model shown in Figure 5 and then, calculation result is $\mathrm{C}=0.5$. The two sources in Figure 5 have the same contamination density.

$$
C=D_{1} / D_{2}
$$

$D_{1}$ : dose at $1 \mathrm{~cm}$ height from the source

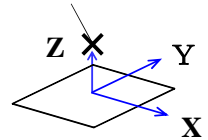

plane source

$(1 \mathrm{~m} \times 1 \mathrm{~m}$ square $)$
$D_{2}$ : dose at $1 \mathrm{~cm}$ height from the source

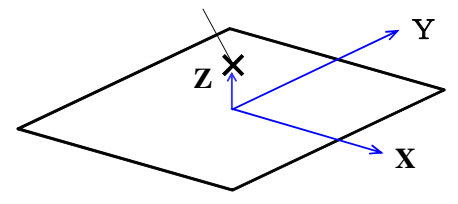

plane source $(100 \mathrm{~m} \times 100 \mathrm{~m}$ square $)$
Figure 5. Calculation model used to develop $C$.

Table 3. Calculation conditions to develop C.

\begin{tabular}{|l|l|}
\hline Calculation code & MCNP5 \\
\hline Shape of source & $\begin{array}{l}2 \text { types of plane sources } \\
(1 \mathrm{~m} \times 1 \mathrm{~m}, 100 \times 100 \mathrm{~m} \text { square } \\
\text { plane source whose strength is } \\
\text { homogeneously distributed })\end{array}$ \\
\hline Radionuclide & $\begin{array}{l}4 \text { types } \\
(\mathrm{Cs}-134, \mathrm{Cs} 137, \mathrm{Co}-60, \\
1 \mathrm{MeV} \gamma \text {-ray })\end{array}$ \\
\hline
\end{tabular}

\section{RaF-MAP verification}

The calculated doses using RaF-MAP were compared to those calculated using QAD[3] to verify the accuracy of the built-in data.

\subsection{Verification of the attenuation rate in the air}

To verify the accuracy of $A(L)$, the doses calculated using RaF-MAP were compared to those calculated using QAD with the model shown in Figure 6. The model has a plane source $(1 \mathrm{~m} \times 1 \mathrm{~m}$ square $)$ with its source strength adjusted to be $10 \mathrm{mSv} / \mathrm{h}$ at $1 \mathrm{~cm}$ from the source. There is no shield in this model and the calculation points are located within the range 0 to $49.5 \mathrm{~m}$ in vertical and horizontal directions relative to the plane source at intervals of $10 \mathrm{~m}$. In this calculation, doses calculated using Raf-MAP were divided by $C$ to eliminate the effect of $C$.

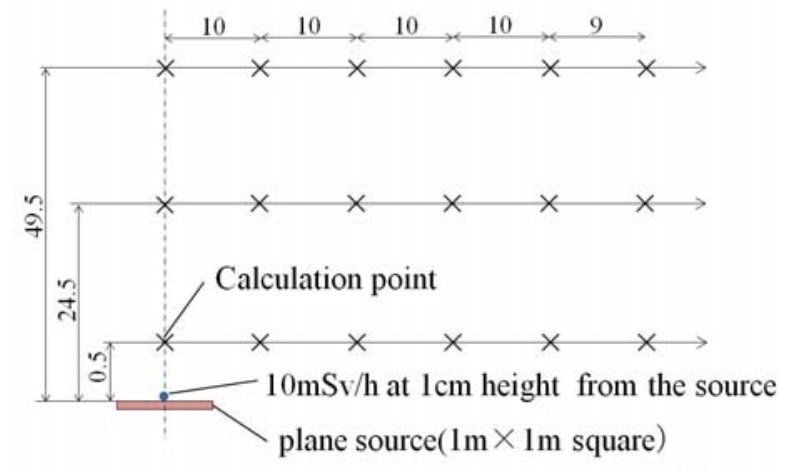
unit:(m)
Calculation results obtained using RaF-MAP shown in Figure 7 were confirmed to reproduce the doses calculated using QAD code within the range +0 to $10 \%$. The differences in the calculation results between RaF-MAP and QAD are expected to be attributable to the following:

- The approximation included in the Eq. (3).

- The lack of angular dependency of $A(L)$ due to the calculation point of basic data being limited to a vertical direction (in Figure 2).

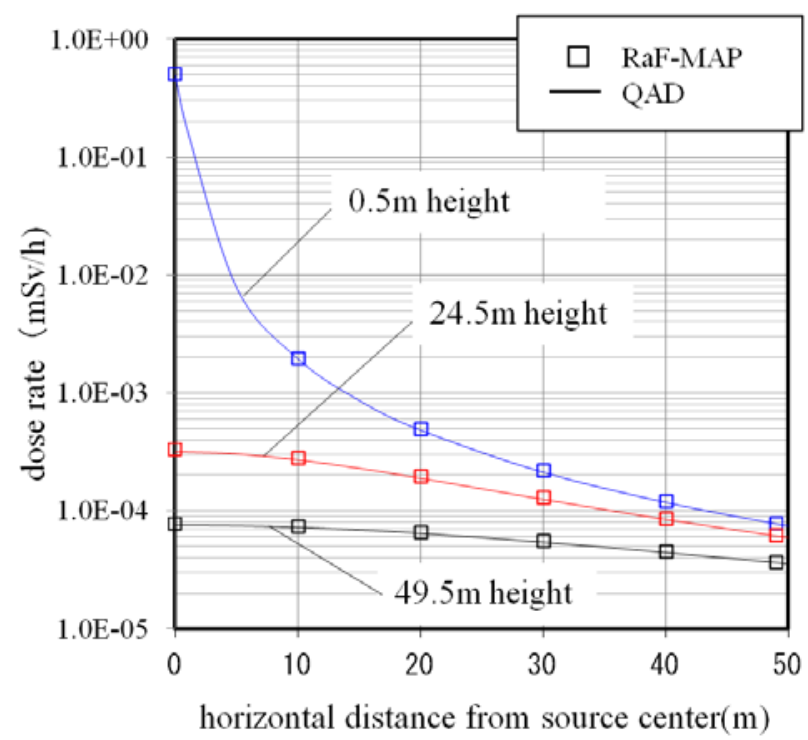

Figure 7. Comparison of doses calculated using RaF-MAP with those calculated using QAD.

\subsection{Verification of attenuation rate in shields}

To verify the accuracy of $R(t)$, doses calculated using RaF-MAP were compared to those calculated using QAD with the model shown in Figure 8. The model has a plane source $(1 \mathrm{~m} \times 1 \mathrm{~m}$ square) whose strength is adjusted to be $10 \mathrm{mSv} / \mathrm{h}$ at a $1 \mathrm{~cm}$ height point from the source. A concrete or steel shield is set parallel to the plane source in this model and the calculation points are located beyond the shield from the plane source $(1 \mathrm{~m} \times 1 \mathrm{~m}$ square). As an example, the calculation results when the shield material is set to concrete are shown in Figure 9.

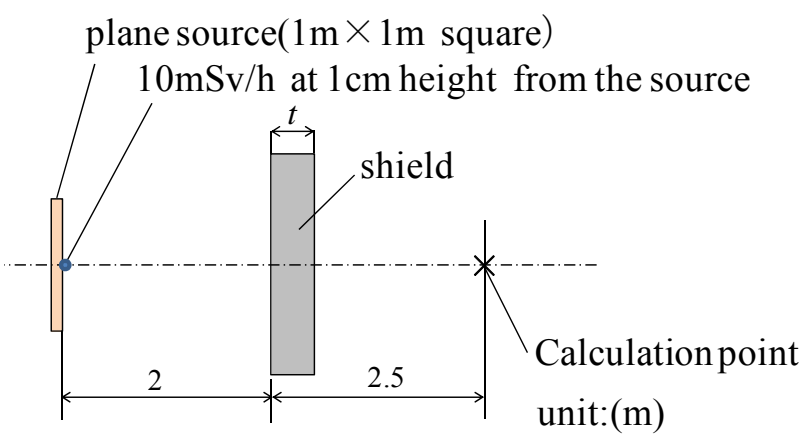

Figure 8. Calculation model to verify the accuracy of $\mathrm{R}(\mathrm{t})$.

Figure 6. Calculation model used to verify the accuracy of $A(L)$. 


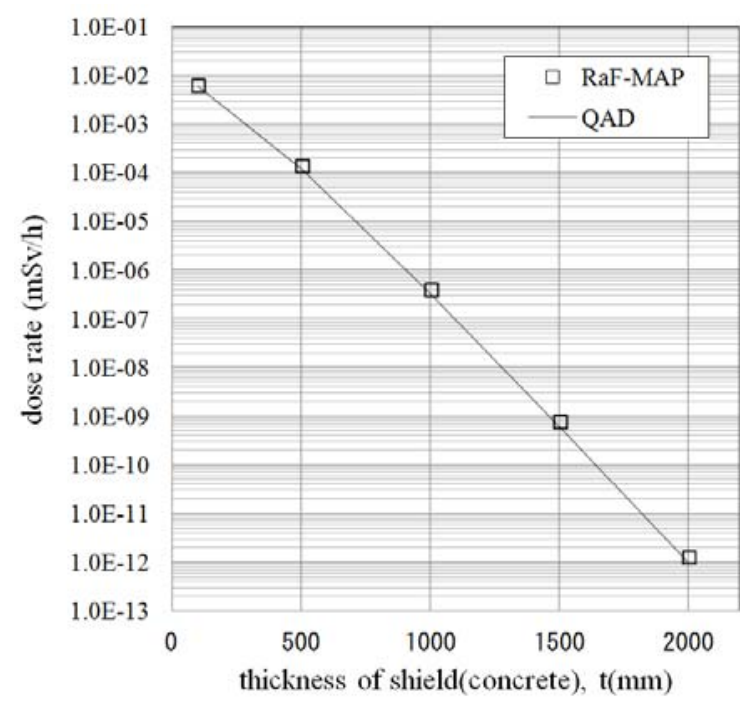

Figure 9. Comparison of doses in shield calculated using RaF-MAP with those calculated using QAD.

The calculation results obtained using RaF-MAP were confirmed to reproduce the doses calculated using QAD code within the range +0 to $30 \%$. The differences in calculation results between RaF-MAP and QAD are expected to be attributable to the following:

While $\gamma$-ray build-up factors in RaF-MAP are based on the assumption of a spherical shield shape, the shape of the shield in this calculation (Figure 8) is flat.

\section{Application to the reactor building at 1F-Unit3}

Doses calculated using RaF-MAP were compared to those measured in the reactor building at $1 \mathrm{~F}$-Unit3. The doses measured in the reactor building are posted on TEPCO's website [4]. The calculations were conducted using the dose measured at the surface of structures as input. The scope of the calculation model was limited to the first floor in the reactor building. The areas considered to be radiation sources in the input of RaF-MAP include the whole surface of the ceiling, the whole surface of the walls, and the surface of the floor yellow-colored in Figure 10. If a mesh has no measured dose to input, the average dose at the adjacent 8 meshes is adopted to enter.

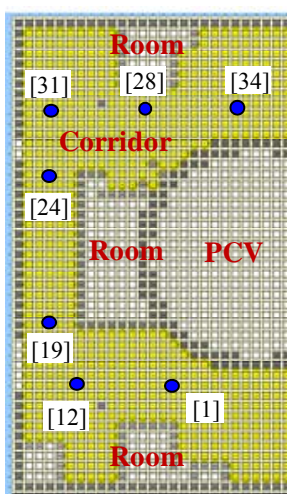

Figure 10. Areas considered radiation sources in the input and the location of the measurement point.
The radiation contour maps calculated at heights of $0.5 \mathrm{~m}$ and $1.5 \mathrm{~m}$ are shown in Figure 11. In addition, the calculated doses at points in Figure 10 at a height of $1.5 \mathrm{~m}$ are listed in Table 4 with measured doses at the same location and ratio as these two values (C/M: Calculated value / Measured value).

$C / M$ s range from 0.4 to 0.9 . The causes of $C / M$ s going under 1.0 are expected to be the following:

- Contamination on the equipment and pipes was not modeled as the radiation source.

- Some hotspots were not modeled.

Considering the causes above, $C / M$ s going under 1.0 are expected to be appropriate tendency. Some hotspots were confirmed by a gamma camera to be on the ceiling near the point [19] whose $\mathrm{C} / \mathrm{M}$ was minimum value among 33points though these hotspots have not reflected to $\mathrm{C} / \mathrm{M}$ due to the difficulty of quantitative analysis. As a reference data, the average of $\mathrm{C} / \mathrm{Ms}$ at 33 points is 0.7 and this means that the difference between the measured and calculated values is almost comparable to the measurement error.

Therefore, exhaustively surveying hot spots using a gamma camera and reflecting these data as input should be effective in raising $C / M$ to 1.0 .

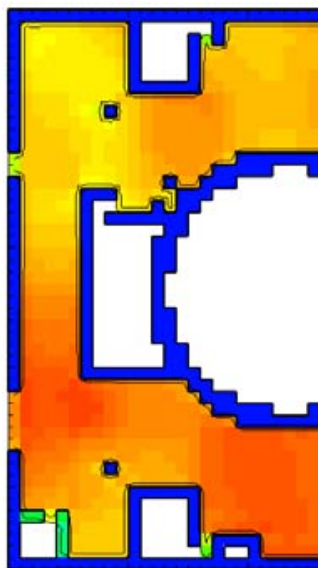

(a) at height of $0.5 \mathrm{~m}$

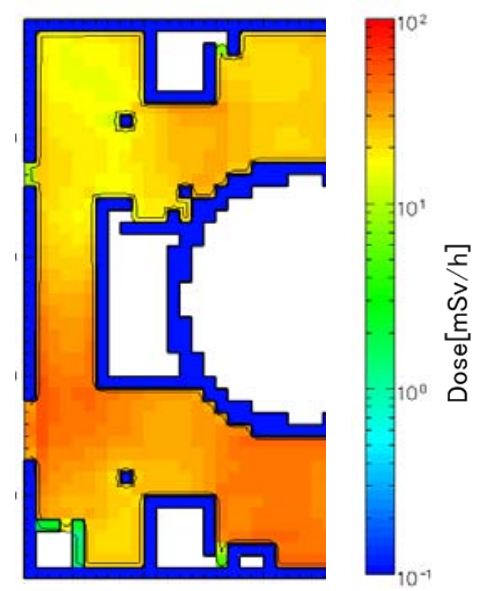

(b) at height of $1.5 \mathrm{~m}$
Figure 11. The radiation contour maps calculated at heights of $0.5 \mathrm{~m}, 1.5 \mathrm{~m}$ from the $1 \mathrm{st}$ floor in $1 \mathrm{~F}-$ Unit 3 reactor building.

Table 4. Comparison of the calculated dose rate at a height of $1.5 \mathrm{~m}$ using an RaF-MAP with a measured value and $C / M$.

\begin{tabular}{|c|c|c|c|}
\hline \multirow{2}{*}{ Point } & \multicolumn{2}{|c|}{ Dose rate } & \multirow{2}{*}{ C/M } \\
\cline { 2 - 3 } & at $1.5 \mathrm{~m}$ height $(\mathrm{mSv} / \mathrm{h})$ & \\
\cline { 2 - 3 } & Measured value & Calculated value & \\
\hline$[1]$ & 44.7 & 38.2 & 0.9 \\
\hline$[12]$ & 53.8 & 33.2 & 0.6 \\
\hline$[19]$ & 98.7 & 41.3 & 0.4 \\
\hline$[24]$ & 21.4 & 19.2 & 0.9 \\
\hline$[28]$ & 35.5 & 25.8 & 0.7 \\
\hline$[31]$ & 34.1 & 18.8 & 0.5 \\
\hline$[34]$ & 37.5 & 22.2 & 0.6 \\
\hline \hline Ave. & - & - & $\mathbf{0 . 7}$ \\
\hline
\end{tabular}

Note: The calculations were performed at 33 points. Table 4 shows an excerpt, which includes Max. and Min. values of 33 $\mathrm{C} / \mathrm{Ms}$. The average in Table 4 is an average of $33 \mathrm{C} / \mathrm{Ms}$. 


\section{Procedure to explore reasonable decontamination conditions with RaF-MAP}

The procedure to explore reasonable decontamination conditions with RaF-MAP is defined as 8 steps as shown in Figure 12. After the calculated doses at points in air are confirmed as consistent with the measured doses, decontamination conditions are set in STEP6, whereupon doses after decontamination are calculated in STEP7. If the calculated doses after decontamination do not correlate with the target criteria dose, the decontamination conditions should be redefined (return to STEP6) and repeat it until the target criteria dose is satisfied. If the calculated doses after decontamination are confirmed as satisfying the target criteria dose, the decontamination conditions set in STEP7 would be considered appropriate.

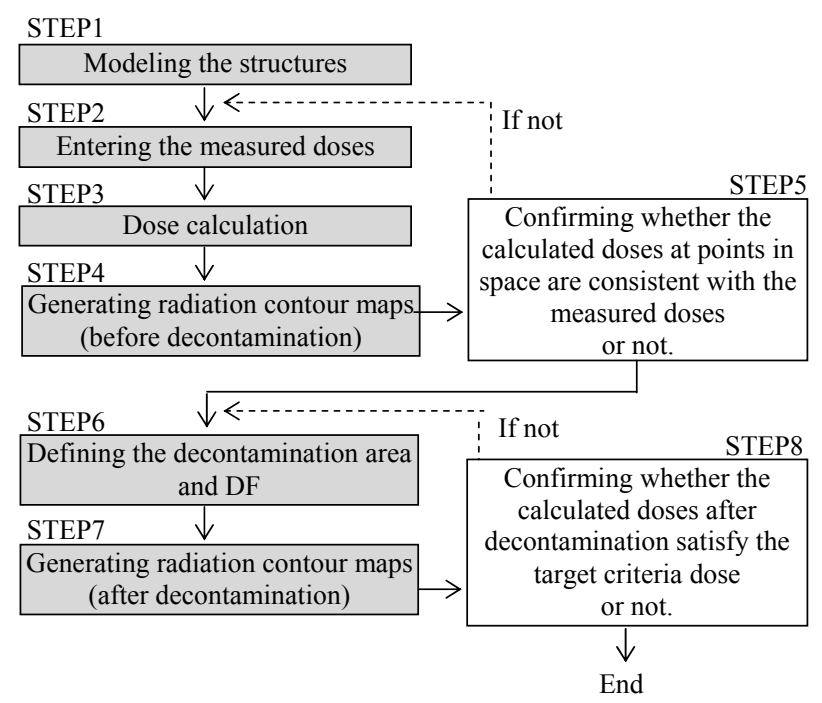

Figure 12. Procedure to explore reasonable decontamination conditions with RaF-MAP.

As an example, Figure $\mathbf{1 3}$ shows the radiation contour maps generated by setting the following conditions of the hypothetical decontamination operation:

- Decontamination area: surface on the floor enclosed

- Decontamination factor: $10^{* 1}$

by a dotted line in Figure 13(a)

*1:Contamination density on the surface after decontamination is reduced to $1 / 10$ of that before decontamination

In this case, the doses at $\mathrm{P}_{1}(1.5 \mathrm{~m}$ height $)$ after decontamination was reduced to 0.7 times than before decontamination.
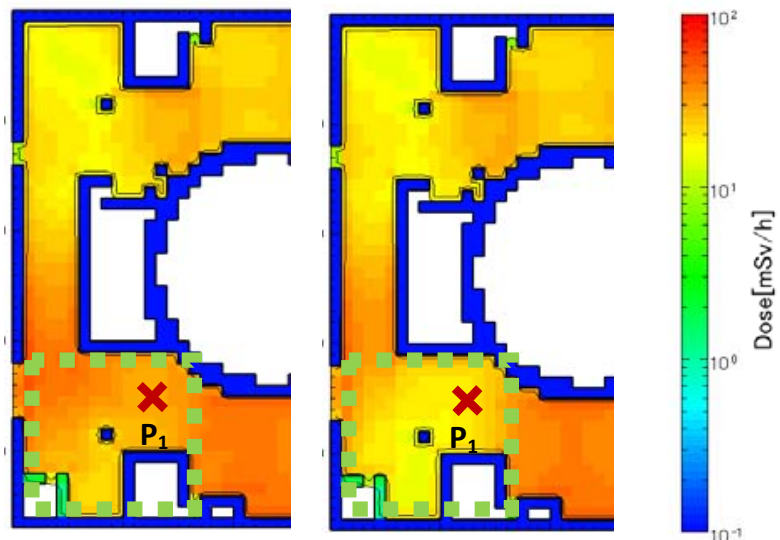

(a) Before decontamination (b) After decontamination

Figure 13. Evaluating the dose-reduction effect achieved by the hypothetical decontamination operation.

\section{Conclusion}

(1) A new simulation system, called "RaF-MAP", calculating the dose result based on contamination on the surface of structures was developed.

(2) Calculation results obtained using RaF-MAP were confirmed to reproduce the doses calculated using QAD code within the range +0 to $30 \%$. Considering this variance is almost equivalent to the measurement error, the specifications of RaF-MAP should be reasonable.

(3) Calculation results using RaF-MAP were confirmed to reproduce the measured doses in the $1 \mathrm{~F}$-Unit3 reactor building about $-30 \%$ on average of 33 points.

(4) The dose-reduction effect of the decontamination operation can easily be evaluated using RaF-MAP. Therefore, RaF-MAP can help determine the decontamination plans.

\section{References}

[1] X-5 Monte Carlo Team, MCNP - A General Monte Carlo N-Particle Transport Code, Version5, LA-UR-03-1987, Los Alamos National Laboratory, (2003).

[2] Y. Harima, H. Hirayama, Y. Sakamoto, N. Sasamoto, F. Masukawa, H. Nakashima, K. Hayashi, H. Handa, R. Tayama, N. Kurosawa, M. Nemoto and T. Abe, Validity of the four-parameter empirical formula in approximating the response functions for gamma-ray, neutron, and secondary gamma-ray skyshine analysis, J. Nucl. Sci. Technol. 40 (2003), pp. 569-578.

[3] R.E. Malenfant, QAD:A Series of Point-Kernel General-Purpose Shielding Program, LA-3573, Los Alamos National Laboratory, (1966).

[4] http://www.tepco.co.jp, handouts_120705_01-j. 